\title{
Adaptation of a double swivel for cross-transfusion
}

\author{
JACOB BRANDSTAETTER and JOSEPH TERKEL \\ Department of Zoology, The George S. Wise Center for Life Sciences, \\ Tel-Aviv University, Tel-Aviv, Israel
}

\begin{abstract}
A system is described for connecting a double lumen chronic heart cannula from a freely moving laboratory animal to a pair of stationary outlets. This system allows the connection of two flow-wavs to the blood system of a stress-free animal. By using two of these devices, a pair of animals can be connected for continuous and rapid blood exchange. It should prove useful in behavioral, psychological and physiological studies. The system can also be adapted for multichannel electric connections and does not stress or disturb the animal.
\end{abstract}

The need for a swivel joint with more than one lumen arose while working on a method for quick exchange of blood between two unrestrained animals. Single lumen swivel joints have been used in a number of systems in recent years, mainly for infusion of substances into the circulation (Epstein \& Teitelbaum, 1962; Thomas \& Mayer, 1968).

When single lumen swivel joints were used in cross-transfusion between freely behaving animals (Terkel, 1972), blood exchange was not sufficiently rapid for study of blood-borne substances with relatively short half lives. When two-channel cross-transfusion was carried out in the absence of a double swivel, it was necessary to restrain or anesthetize the subjects (Brodish \& Long, 1956; Weinstein \& Annau, 1967). Thus, a two-way double swivel for rapid blood exchange between freely moving animals has obvious advantages over existing systems for behavioral and physiological studies.

Two-channel or multichannel swivel joints found in industry are not designed to take into account the unusual fluid properties of blood and the problems encountered in animal movement. The usual type of double swivel consists of a pair of abutting members which rotate relative to each other. Both have central recesses that together define a chamber. This contains a pair of concentrically positioned o-rings that divided the chamber into a central circular region and an outer annular region. Two axial apertures in each member connect with the respective regions of the chamber. When the system is axially pressed together, the two regions of the chamber are sealed from each other and from the exterior by the o-rings. We were able to miniaturize such a device, but found that the movement of the animal did not provide enough force through its connecting conduit to rotate the swivel. To overcome

We are indebted to Professor Bruce Rothschild, Mathematic Department, University of California at Los Angeles, for critical reading of the manuscript, and to $Z$. Eisner and $A$. Shubb for preparation of the photographs. This study was supported in part by the Fund for the Encouragement of Research of the Histadrut-General Federation of Labor of Israel. this problem we introduced a servomechanism to rotate the device. A second problem lay in the fact that a slack conduit could become accessible to gnawing by the experimental animals. Suspending the system by means of a counterbalance, as with a single lumen swivel (Terkel, 1972), imposes unnatural loads on the animal because of the high inertia of the apparatus. This problem was solved by adding a second servomechanism. It raises and lowers the mechanism according to the

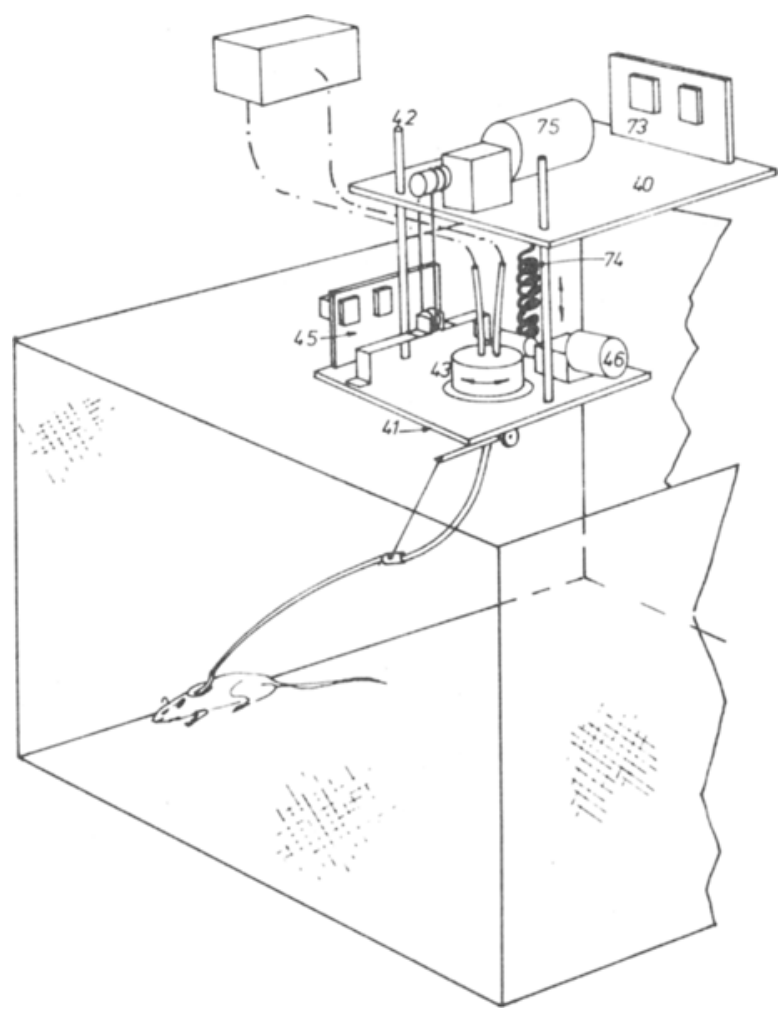

Figure 1. General layout of the system. The experimental animal is connected by conduit leading the double cannula to the swivel joint, on Base 41 . The base is suspended on cable and is free to move up and down. Details are included in the text. 

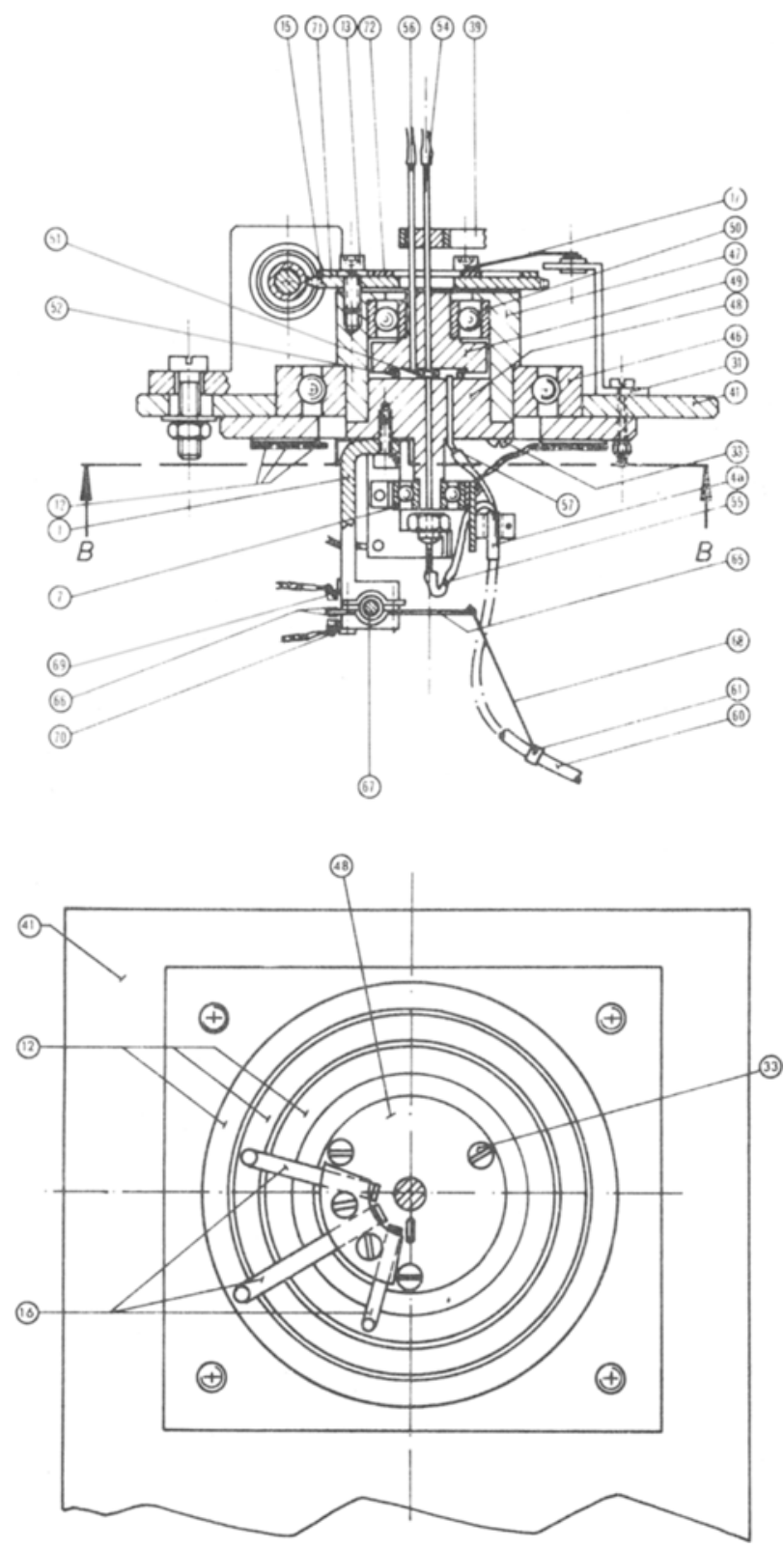

Figure 2a. A cross section of the swivel joint and the sensors. The rotating part of the swivel, Cup 47 and Plug 48 is anchored in Ball Bearing 46. The sensors are mounted on the plug's lower face. Between the stationary part, Flange 49 and Plug 48, the two seals, 51 and 52 , are seen.

Figure 2b. Bottom view of Figure 2a (Section BB) showing the slip rings, 12, resting on the lower face of Base 41 . The wiper assembly, 16, is connected to Plug 48 .

tension or slack on the conduit.

Figure 1 shows the general layout of the system. The stationary frame, 40 , is rigidly fixed relative to the cage. A base, 41 , carries a pair of guide rods, 42 , slidably supported in suitable busings (not shown) in Frame 40, so that the base can move up and down. The servomechanism controlling rotation consists of a motor,
46, moving the swivel, 43 , and controlled by the electronic components, 45 .

The experimental animal (a rat) is cannulated via the right jugular, the cannula tip resting in the right atrium and the free end of the cannula emerging at the nape of the neck to connect with the swivel system. The present cannulation procedure is identical to that described by Terkel (1972), except that double lumen expanded cannula (Dural Plastics and Engineering, Dural N.S.W. 2158 Australia; Catalog DVE 4) is used instead of single lumen tubing (for details of the surgical procedure see Terkel, 1972).

Figure 2 a shows a section through the base, 41 , and the swivel joint. The swivel joint cup, 47 , is attached to the inner race of Ball Bearing 46 , the outer race of which is rigidly attached to the base, 41 . Cup 47 and Plug 48 can rotate $360 \mathrm{deg}$. The nonrotating portion of the swivel consists of an enlarged flange, 49 , whose outer diameter is smaller than the inner diameter of Cup 47. A hub projects upward from the flange, carrying the inner race of Ball Bearing 50, by which the rotary portion of the swivel may rotate relative to 49 . The lower face of Flange 49 is provided with a recess into which the outer o-ring, 51, is fitted (Abscoa Industries, Inglewood, California part 2-12 T). The inner o-ring, 52 (Abscoa Industries, part 2-004 T), is held in place by the central stainless steel tube (19 ga), 54, which protrudes into the chamber that is defined by Plug 48 and the lower face of Flange 49.

A central circular region is defined by O-Ring 52, and an annular canal by the outer o-rings. Axial pressure is provided by three screws, 33, (Figure 2a and b) that hold the plug in place under Cup 47. Unbroken fluid flow is thus assured regardless of the angular position of the movable part with respect to the stationary part of the swivel. The central tubes, 54 and 55 , and the outer ones, 56 and 57, create two unbroken flow-ways. The stationary part (Flange 49 and Tubes 54 and 56) is held against rotation by Bracket 39 , which is fixed to Base 41.

Animal movements are detected both by a torque sensor and by a tension sensor, mounted on Frame 1 (Figure 2a and Figure 3). Torque is transmitted along a tough conduit, 60 , that protects the double lumen tube. The conduit, 60, terminates in Clamp 4 (Figure 3a). The flexible double lumen tube emerges from Conduit 60 terminating in Tubes 55 and 57 (Figure 2a). The clamp is soldered to Extension Arm 4a (Figure 3b) which carries two contact terminals. The arm is rigidly attached to Ball Bearing 7 (Figure 2a and Figure 3a) and is free to move in relation to Plug 48 . Every small rotary movement by the animal induces slight twisting of the conduit, resulting in a small angle rotation of Arm 4a. This in turn closes one of the contacts between $4 \mathrm{a}$ and 5 (Figure $3 b$ ), activating a relay in the rotary servo-system The relay main circuit is then closed, and a two-way electromotor rotates the swivel in the correct direction by means of a worm gear which mates with Worm Wheel 

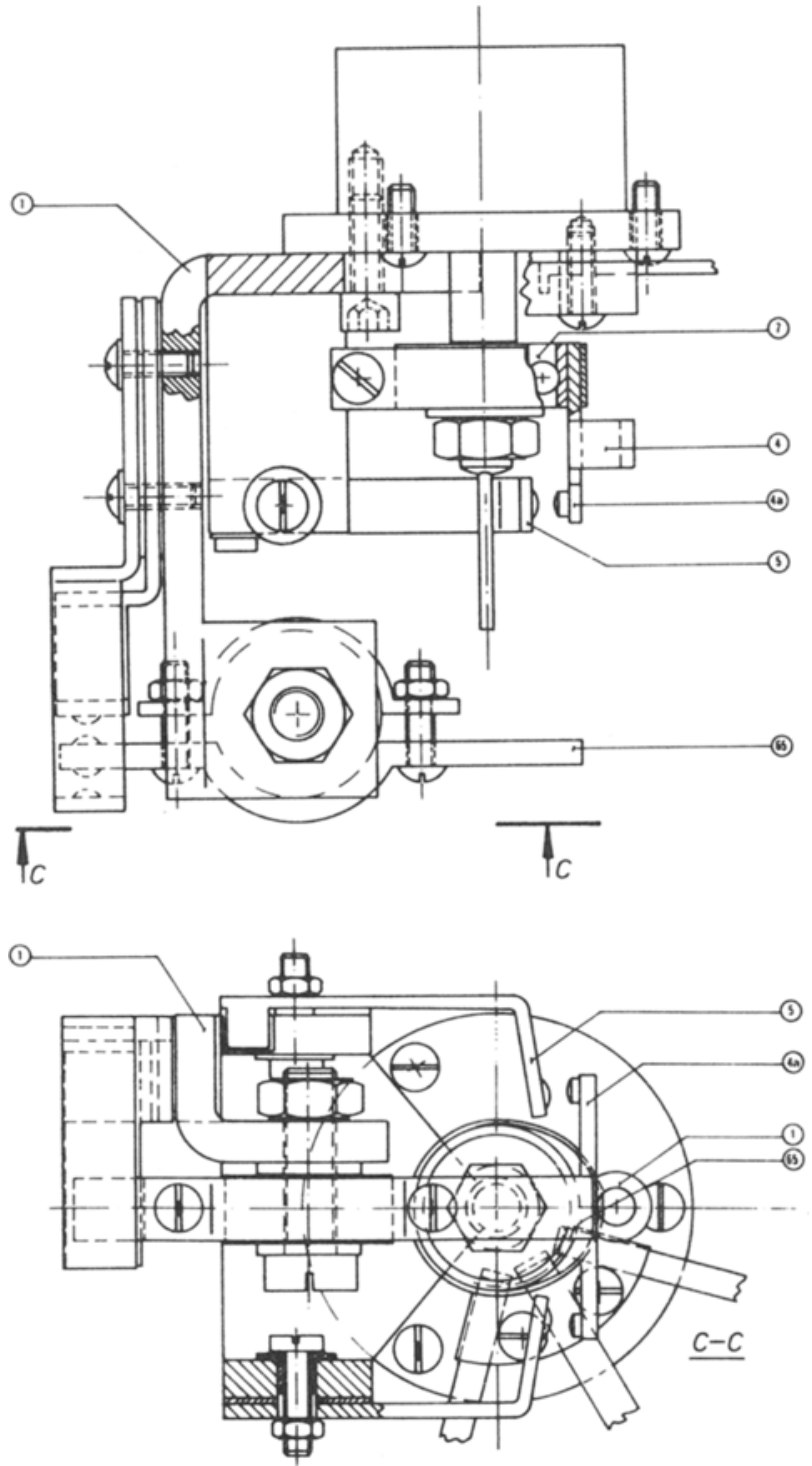

Figure 3a (Above). Detailed drawing of the sensors. Clamp 4 and Extension-Arm $4 \mathrm{a}$ are mounted on Ball Bearing 7, and are free to rotate and close the contact between $4 \mathrm{a}$ and 5 . The up and down sensor is shown with Arm 65 mounted on its pivot.

Figure 3b (Below). Bottom view. The relative position of Arm $4 a$ and Contact 5 is clearly shown.

15 (Figure $2 \mathrm{a}$ ) rigidly attached to Cup 47 . Rotation of the swivel continues until the starting position relative to the animal is reached (i.e., zero torque), and the contact between $4 \mathrm{a}$ and 5 is broken. Continuous contact, regardless of angular position, between the sensor on the rotating part and the relays and power supply on Base 41 is effected by a three-finger wiper assembly, 16 (Figure 2b), each member of which carries a contact slidably engaged with respective Slip Rings 12 (Figure 2a and Figure $2 \mathrm{~b}$ ). The servo-motor is high speed dc capable of 3000 RPM reduction through worm gear with a ratio of $1 / 80$; this gives a final swivel speed of 37.5 RPM which is sufficient to follow the movement of a rat.

The tension sensor consists of a rigid arm, 65 (Figure 2a), pivoting on Bushing 67. The free end of the arm is connected through Link 68 to Clamp 61 on the conduit. The opposite end of the arm is provided with contacts that may engage with one of the two separate Contacts 69 and 70 . Contacts 69 and 70 are connected by means of insulated wires to Slip Rings 71 and 72 mounted on Worm Wheel 15 (Figure 2a). (The wires pass through a channel drilled through Cup 47 and the worm wheel, 15, to end in a soldered connection on the slip rings). Continuous connection between Slip Rings 71 and 72 and the vertical servo-circuit is assured by the two-finger Wiper assembly 17 (Figure 2a) rigidly mounted on Base 41 by means of Support 31. The fingers are connected through self-coiling insulated telephone wire 74 (Figure 1) with the relay coil terminals. The relays are attached to the Electronics Board 73 on Frame 40 (Figure 1).

Lateral movement of the animal in the cage lowers Arm 65 (Figure 2a) so that the contact end ascends and the contact on Arm 65 is engaged. Arm 65 is attached to Sliding Contact 16 which is connected through Slip Ring 12 to the positive pole of a battery. The engagement of the contact on Arm 65 closes the coil circuit of the down command relay, and Motor 75 (Figure 1) rotates, unwinding the flexible cable from which Base 41 is suspended. The lowering continues until the tension on the conduit is reduced and the contact returns to neutral position. Movement of the animal back to the center causes slight slackening of the conduit. The contact end of Arm 65 is lowered by means of $1 . \mathrm{g}$ weight. Contact 70 is engaged, and the servo-motor winds the cable up and Base 41 ascends until neutral position is reached.

The electric $\mathrm{dc}$ is supplied by a heavy duty $12 \cdot \mathrm{V}$ battery. Each of the two command circuits (one for

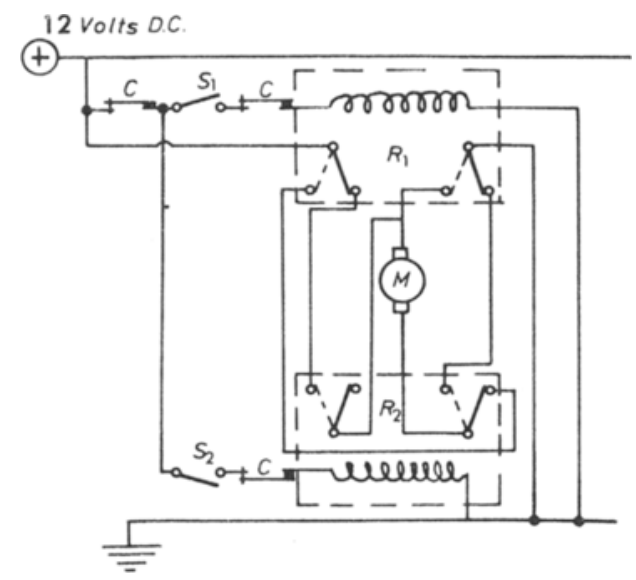

Figure 4. Switching diagram of the circuit which is used in the rotary and the up and down. R-DPDT leaching relay, S-sensor's switch. C-sliding contact. M-motor. 
vertical and one for rotary movements, Figure 4) consists of a pair of double pole double throw latching relays and a motor. Closing one relay circuit rotates the motor in one direction, while closing of the other reverses the polarity on the motor terminal, and rotates it in the reverse direction. The sensors control the relay switching by closing or opening of the relay coil circuits.

A prototype of the double swivel joint system described above has been built and operated in our laboratory. The system has been tested and is suitable for behavioral studies.

In designing the system, we took care to use standard components. The only custom made parts are the swivel joint and the sensors. The mechanical parts are standard components manufactured by companies such as PIC Design Corporation in USA, and Reliance Gears Company Limited, in England. Electromotors and the relays are available in every electronic supply store. It is important to use only first class materials for the sensor contacts, as they tend to oxidize readily.

\section{REFERENCES}

Brodish, A., \& Long, C. N. H. A technique of cross circulation in the rat which permits accurate control of blood volume transfers. Yale Journal of Biology and Medicine, 1956, 28, 644-656.

Epstein, A. N., \& Teitelbaum, P. Watertight swivel joint permitting chronic injection into moving animals. Journal of Applied Physiology, 1962, 17, 171-172.

Terkel, J. A chronic cross-transfusion technique in freely behaving rats by use of single heart catheter. Journal of Applied Physiology, 1972, 33, 519-522.

Thomas, D. W., \& Mayer, J. Simple and inexpensive swivel joint for the infusion of unrestrained animals. Physiology and Behavior, 1968, 3, 499-500.

Weinstein, S, A \& Annau, Z A chronic cross circulation technique for rats. Journal of Applied Physiology, 1967, 23, 601-604. 